\title{
Inferring the impact of linguistic boundaries on population differentiation: application to the Afro-Asiatic-Indo-European case
}

\author{
Isabelle Dupanloup de Ceuninck ${ }^{1}$, Stefan Schneider ${ }^{1}$, André Langaney ${ }^{1,2}$ and \\ Laurent Excoffier ${ }^{1}$ \\ ${ }^{1}$ Laboratoire de Génétique et Biométrie, Département d'Anthropologie, Université de Genève, Geneva, Switzerland; \\ ${ }^{2}$ Laboratoire d'Anthropologie Biologique, Musée de I'Homme, Paris, France
}

We present here a quantitative way to assess the impact of language-family boundaries on population differentiation and to evaluate the homogeneity of the genetic processes along these boundaries. Our estimator ( $\delta a)$ of the impact of the boundary is based on an isolation by distance (IBD) model and measures the added genetic distance between populations located on different sides of the boundary. We compare this statistic with another estimator of group differentiation $\left(F_{C T}\right)$ computed under an analysis of variance framework that does not assume any particular spatial structure of the populations. Monte Carlo simulations are used to study the behaviour of these statistics under a two-dimensional stepping-stone model. Simulations show that $\mathrm{F}_{\mathrm{CT}}$ can suggest the existence of a frontier when populations only differ because of IBD. This spurious behaviour is much less frequent for the da statistic. However, the large variance associated with the $\delta a$ statistic, and the fact that it should only be computed in the presence of IBD, may limit the use of this statistic. Overall, the origin and the effect of the boundary is best understood by comparing different statistics and by testing for the presence of IBD on each side of the boundary as well as across the boundary. We illustrate our approach by examining the boundary between Afro-Asiatic and Indo-European populations. These populations are globally genetically differentiated, but the effect of the linguistic boundary on gene flow seems geographically very heterogeneous. This boundary appears to be the result of a secondary contact between two differentiation centres rather than an enhancer of population differentiation. European Journal of Human Genetics (2000) 8, 750-756.

Keywords: human populations; linguistic boundaries; gene flow; isolation by distance; Monte Carlo simulations

\section{Introduction}

Under the isolation by distance (IBD) model, ${ }^{1-3}$ one would expect measures of genetic differentiation at neutral loci to increase with geographic distances. Although this model usually fits short-range migrations between neighbouring populations, the covariation of geographic and genetic distances is revealed by most analyses of world and continental population data. ${ }^{4-9}$ Several studies have indeed revealed a strong correlation between the linguistic and the

Correspondence: Dr Isabelle Dupanloup de Ceuninck, Laboratoire de Génétique et Biométrie, Département d'Anthropologie, Université de Genève, Case Postale 511, 1211 Genève 24, Switzerland.

Tel: +4122702 6940; Fax: +41 223000351 ;

E-mail: isabelle.dupanloup@anthro.unige.ch

Received 7 October 1999; revised 2 May 2000; accepted 3 May 2000 genetic differentiation of human populations on a worldwide $^{10,11}$ or on a continental ${ }^{6,12,13}$ scale. In the spatial study of genetic polymorphism, regions of sharp genetic changes often correspond to physical barriers, ${ }^{14}$ language boundaries, or to both, indicating a departure from a pure IBD model..$^{14-17}$ The association of linguistic boundaries with increased genetic differences could be due to two different processes: the active contribution of the language boundary in preventing gene flow between linguistic groups, or the allopatric differentiation of linguistic groups which then come into contact, creating a zone of sharp cultural and linguistic variation.

Several techniques have been developed to detect the presence of genetic boundaries corresponding to spatial areas 
where the rate of change of gen efrequencies is particularly high. ${ }^{14,18-20}$ When these boundaries are detected, they are often compared with physical or cultural barriers. The goal is usually to interpret the spatial structure of gene frequencies in the light of known ecological or cultural processes.

Several authors have also tried to ascertain the biological impact of linguistic boundaries on the extent of gene flow between populations. ${ }^{15,16}$ The frontiers are here defined a priori and one tests for increased genetic change at these boundaries.

We present here a new approach, specifically based on an IBD model, to assess the genetic impact of linguistic boundaries and to determine if they correspond to significant genetic boundaries. We compare this approach with a more conventional hierarchical analysis of genetic structure without assuming any geographic patterning of the populations. We evaluate the power of our method by Monte Carlo simulations and finally apply our methodology to the case of human populations tested for a classical autosomal marker.

\section{Material and methods}

\section{Determination of the boundary location}

Our methodology does not require one to know the exact location of the linguistic boundary under study. From the geographical location of the sampled points, we construct a set of Voronoi polygons, ${ }^{21}$ which correspond to influence areas around each sampling point (Figure1). The Voronoi diagram is used to find the location of the boundary by searching the edges of the polygons that separate the members of the two linguistic groups.

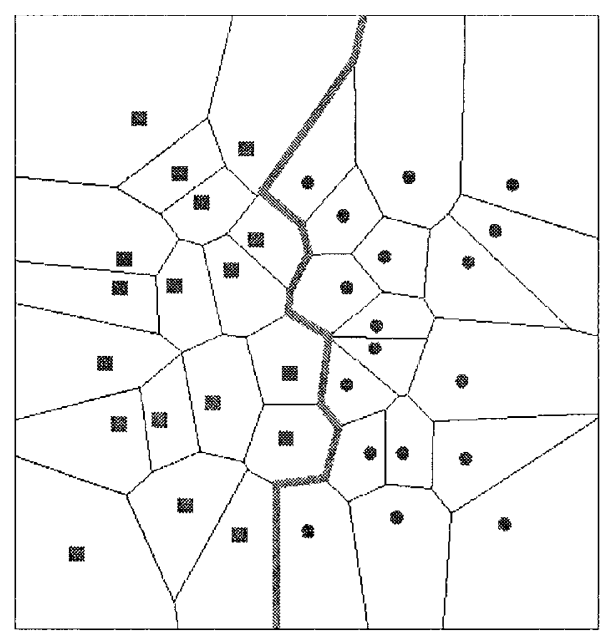

Figure 1 Example of a Voronoi diagram: A plane with $n$ points is partitioned into $n$ convex polygons such that every point within a given polygon is closer to its central point than to the central point of any other polygon.

\section{Segmentation of the boundary}

Because the evolutionary processes acting on different portions of the boundary may be heterogeneous, we can divide the boundary into segments of arbitrary size and then independently evaluate the impact of each segment on genetic differentiation. Some portions of the linguistic boundary may indeed act as strong genetic barriers (eg if they correspond to an ecological frontier or if they somewhat restrict gene flow), and some others may not be associated with enhanced genetic differentiation. The goal of this segmentation process is to understand the fine scale genetic processes at work along the boundary.

As the segmentation criterion, we can choose to divide the whole boundary either into a given number of segments or into segments of fixed length. When the number of segments is fixed, the length of each segment is defined such as having an approximately equal number of population samples associated with it.

\section{Evaluation of the genetic impact of the boundary}

We first test for a genetic difference between groups defined by the boundary, taken as a whole or in segments, using a hierarchical analysis of molecular variance (AMOVA). ${ }^{22}$ The $F_{C T}$ statistic, corresponding to the relative extent of total gen etic variance due to differences between linguistic groups, is estimated from the data and then tested by a nonparametric approach consisting in permuting populations among groups. ${ }^{22,23}$ Our second approach estimates whether populations separated by the boundary show increased genetic distances compared with populations separated by the same geographical distance, but located on the same side of the boundary. An estimator $\delta a$ of the additional intergroup distance is computed from a regression model of genetic distances on geographic distances, and it is tested by a permutation procedure. The model we propose here assumes that population differences are caused by IBD on both sides of the boundary. Therefore, it is necessary to fit an IBD model to each language group. It has been shown ${ }^{24}$ that in a 2D stepping-stone model of population structure where IBD occurs, we should expect an approximately linear relationship between genetic distances computed as $\mathrm{F}_{\mathrm{ST}} /$ $\left(1-F_{S T}\right)$ and the logarithm of the geographic distances between populations. We have thus chosen to proceed further in our evaluation of the impact of the boundary only if the Mantel correlation ${ }^{25}$ between genetics and geography is significant in at least one of the two language groups. Under the null hypothesis of an absence of a boundary effect we would expect similar genetic distances between populations located at a given geographic distance, irrespective of their location relative to the boundary. Therefore, we propose to perform separate regressions of genetic distances on geographical distances at the within-group and at the intergroup levels, and to measure the difference in the $\mathrm{Y}$-axis intercepts of the two regressions as $\delta a=a^{\text {inter }}-a^{\text {intra }}$ (Figure 2 ). 


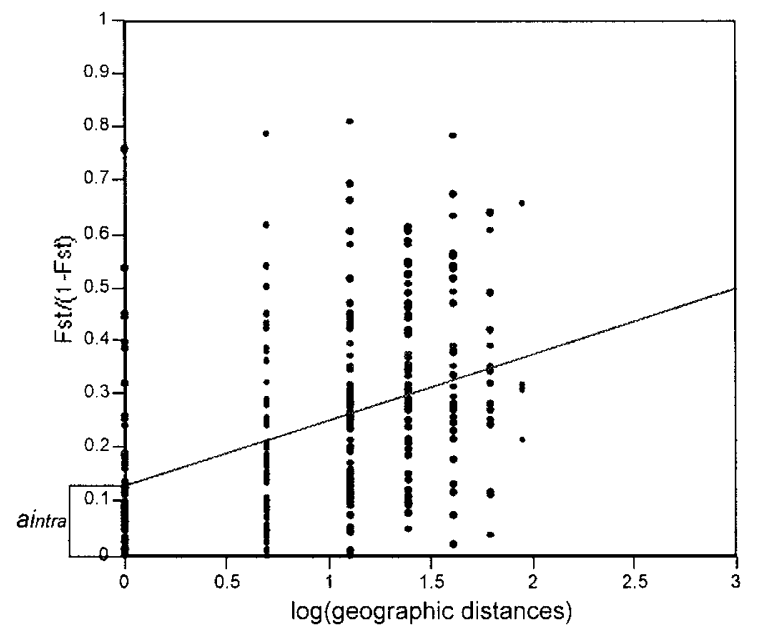

Intra-group regression

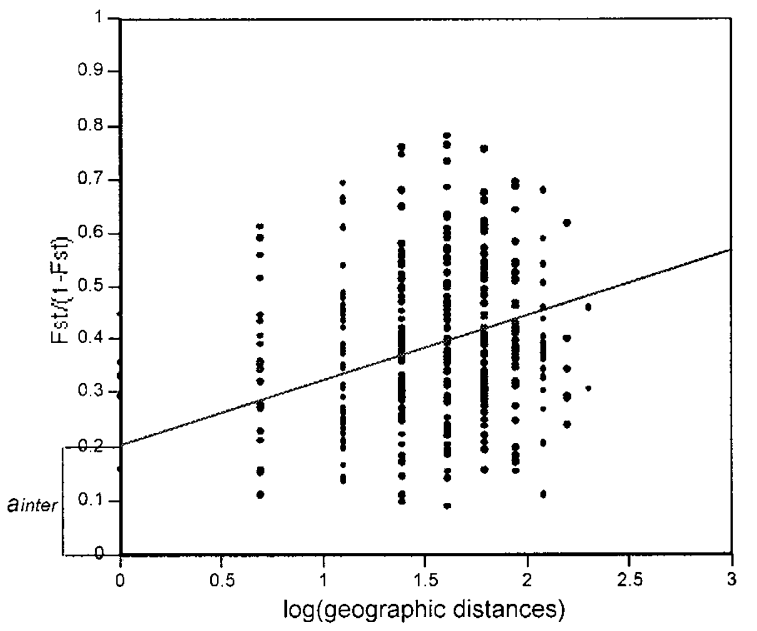

Inter-group regression

Figure 2 Regression of $\mathrm{F}_{\mathrm{ST}} /\left(1-\mathrm{F}_{\mathrm{ST}}\right)$ on the logarithm of geographic distances between populations located on each side of a boundary or a segment of boundary (left: intra-group distances, right: inter-group distances). This example is drawn from a simulation of case 4 in Table1.

Moreformally, we evaluate intra group regression coefficients $b^{\text {intra }}$ and $a^{\text {intra }}$ from the relationship

$$
d_{\text {gen }}^{\text {intra }}=b^{\text {intra }} \log \left(d_{\text {geo }}\right)+a^{\text {intra }}
$$

If the boundary increases the genetic distances between populations on different sides, the inter-group regression coefficient $a^{\text {inter }}$ estimated from the relationship

$$
\mathrm{d}_{\text {gen }}^{\text {inter }}=b^{\text {intra }} \log \left(d_{\text {geo }}\right)+a^{\text {inter }}
$$

will exceed $a^{\text {intra }}$. The $\delta a$ statistic thus directly provides an estimation of the additional genetic distance due to the boundary effect. Statistical significance of the da statistic is evaluated by randomly allocating populations to a given group (a permutation procedure), and recomputing the $\delta a$ statistic after each permutation round in order to get its null distribution. The $P$ value of the $\delta a$ statistic is obtained at the fraction of the cases where a random $\delta a$ value is equal to or larger than the observation.

\section{Simulation study}

We have compared the behaviour of the $\mathrm{F}_{\mathrm{CT}}$ and $\delta$ a statistics by Monte Carlo simulations. Using a coalescent approach, ${ }^{26}$ we have simulated the geneal ogies of two groups of 18 demes arranged on a 2D $6 \times 6$ flat stepping-stone grid. The migration rates between neighbouring demes within a group where uniform and constant in time, and usually larger than the migration rates between neighbouring demes of different groups. The geographic distances between demes were computed as Manhattan block distances on the $2 \mathrm{D}$ grid. Ten DNA sequences of 200 base pairs were simulated for each deme. The size of each deme was fixed to 10000 haploid individuals and the mutation rate to $6 \times 10^{-4}$ per sequence per genera- tion. For each simulated sequence geneal ogy, Poisson-distributed mutations were then introduced on to the realised genealogical tree, starting from the most recent common ancestor of all 360 sequences. Table 1 shows the results of different simulated migration schemes between neighbouring populations to study the effect of IBD on the performance of the $F_{C T}$ and the $\delta a$ statistics. For each set of demographic parameters, we simulated 1000 coalescent processes with mutations in order to get the empirical distribution of the $\delta a$ and the $F_{C T}$ statistics. The significance of the two statistics was further obtained by 1000 permutations of populations between groups for each of the 1000 cases.

\section{Results \\ Simulation study}

The average correlation coefficients between $F_{S T} /\left(1-F_{S T}\right)$ and the logarithm of the geographic distances between populations located on each side of the simulated boundary are given in Table 1, together with their standard deviations and the fraction of cases with evidence for IBD. A pattern of IBD is clearly detected for populations exchanging one or fewer migrants per generation $(\mathrm{Nm} \leq 1)$. For intra-group $\mathrm{Nm}$ values larger than 10, the correlation is low but still significant in slightly more than onethird of the simulations, showing that a geographical structure is maintained despite high levels of gene flow.

The $F_{C T}$ statistic was found significant in almost all the simulations. The $F_{C T}$ mean value increases with the ratio of the intra-group over inter-group migration rates, but it is also dependent on the absolute value of $\mathrm{Nm}$ between groups.

The behaviour of the $\delta a$ statistic is different from that of $F_{C T}$ in several respects. First, its variance is always larger. 
Table 1 Results of the simulation study

\begin{tabular}{|c|c|c|c|c|c|c|c|c|c|c|c|}
\hline $\begin{array}{l}\text { Case } \\
\text { no. }\end{array}$ & $\begin{array}{l}\text { No. } \\
\text { intra- } \\
\text { group }\end{array}$ & $\begin{array}{l}\text { No. } \\
\text { inter- } \\
\text { group }\end{array}$ & $r^{a}$ & Signif. ${ }^{b}$ & $r^{c}$ & Signif. ${ }^{b}$ & $\mathrm{~F}_{\mathrm{CT}}{ }^{\mathrm{d}}$ & Signif. ${ }^{b}$ & $\delta a^{d}$ & $\begin{array}{l}\text { No. } \\
\text { tested } \\
\text { cases }^{\mathrm{e}}\end{array}$ & Signif. $^{f}$ \\
\hline 1 & 10 & 1 & $\begin{array}{c}0.122 \\
(0.110)\end{array}$ & 0.387 & $\begin{array}{c}0.121 \\
(0.109)\end{array}$ & 0.384 & $\begin{array}{c}0.039 \\
(0.022)\end{array}$ & 0.999 & $\begin{array}{c}0.025 \\
(0.024)\end{array}$ & 600 & 0.775 \\
\hline 2 & 10 & 0.1 & $\begin{array}{c}0.117 \\
(0.110)\end{array}$ & 0.380 & $\begin{array}{c}0.114 \\
(0.108)\end{array}$ & 0.363 & $\begin{array}{c}0.192 \\
(0.076)\end{array}$ & 1.000 & $\begin{array}{c}0.245 \\
(0.148)\end{array}$ & 584 & 0.997 \\
\hline 3 & 1 & 1 & $\begin{array}{c}0.308 \\
(0.116)\end{array}$ & 0.913 & $\begin{array}{c}0.301 \\
(0.113)\end{array}$ & 0.914 & $\begin{array}{c}0.046 \\
(0.025)\end{array}$ & 0.952 & $\begin{array}{c}-0.008 \\
(0.050)\end{array}$ & 993 & 0.220 \\
\hline 4 & 1 & 0.1 & $\begin{array}{c}0.321 \\
(0.115)\end{array}$ & 0.942 & $\begin{array}{c}0.320 \\
(0.119)\end{array}$ & 0.931 & $\begin{array}{c}0.164 \\
(0.063)\end{array}$ & 1.000 & $\begin{array}{c}0.186 \\
(0.145)\end{array}$ & 993 & 0.918 \\
\hline 5 & 1 & 0.01 & $\begin{array}{c}0.325 \\
(0.124)\end{array}$ & 0.920 & $\begin{array}{c}0.326 \\
(0.126)\end{array}$ & 0.919 & $\begin{array}{c}0.342 \\
(0.081)\end{array}$ & 1.000 & $\begin{array}{c}0.640 \\
(0.270)\end{array}$ & 995 & 1.000 \\
\hline 6 & 0.5 & 0.05 & $\begin{array}{c}0.309 \\
(0.121)\end{array}$ & 0.922 & $\begin{array}{c}0.313 \\
(0.115)\end{array}$ & 0.918 & $\begin{array}{c}0.193 \\
(0.069)\end{array}$ & 1.000 & $\begin{array}{c}0.241 \\
(0.220)\end{array}$ & 986 & 0.883 \\
\hline 7 & 0.5 & 0.01 & $\begin{array}{c}0.316 \\
(0.123)\end{array}$ & 0.913 & $\begin{array}{c}0.310 \\
(0.122)\end{array}$ & 0.911 & $\begin{array}{c}0.313 \\
(0.076)\end{array}$ & 1.000 & $\begin{array}{c}0.611 \\
(0.302)\end{array}$ & 983 & 0.986 \\
\hline
\end{tabular}

average correlation coefficient between $\mathrm{F}_{\mathrm{ST}} /\left(1-\mathrm{F}_{\mathrm{ST}}\right)$ and the logarithm of geographic distances among populations from the first group. Standard deviation is shown in parentheses; ${ }^{b}$ Fraction of correlations significant at the $5 \%$ level, out of 1000 simulated cases; ${ }^{C} A{ }^{a}$, but applies to second group; dStandard deviation is shown in parentheses; 'Number of cases out of 1000 where the significance of the $\delta a$ statistic was tested, because the hypothesis of IBD within-group was retained in at least one of the two groups; ${ }^{\mathrm{f}}$ Fraction of significant cases at the $5 \%$ level, out of the number of tested cases shown in the previous column.

Second, when $\mathrm{Nm}=1$ (cases 1 and 3), $\delta a$ appears to perform better than $\mathrm{F}_{\mathrm{CT}}$ in the sense that its average value is low, and the number of significant tests is much lower than for $\mathrm{F}_{\mathrm{CT}}$, as it should be for a weak barrier to gene flow. It is interesting to note that in the absence of any boundary (case 3 ), $F_{C T}$ still indicates a clear differentiation of the two groups, which wrongly suggests the presence of a significant boundary. This counter-intuitive result is only due to the effect of IBD: populations from different groups are indeed genetically more divergent because they are farther apart. The da statistic shows here much less significant cases $(22 \%)$ than $F_{C T}$ (95.2\%), because it takes IBD into account, whereas $F_{C T}$ does not.

\section{Application to human populations of Eurasia and Africa} We have studied the linguistic boundary between AfroAsiatic and Indo-European populations tested for the serological RH system. We have used the RH system despite its low level of polymorphism because it has been tested in many populations and thus provides a quite uniform spatial distribution of the population samples of the two linguistic groups under investigation.

We have selected 58 Afro-Asiatic and 100 Indo-European samples for the present study (Figure3) from a worldwide database containing about 600 population samples tested for the RH system. ${ }^{27}$ We have arbitrarily divided the linguistic boundary into five segments. Our segmentation choice is a compromise between the division of the boundary into very small portions which would allow study of finescale genetic processes along the boundary, and the need for a sufficient number of populations associated with each segment in order to be able to perform meaningful statistical analyses.
A pattern of IBD is observed for most groups of populations located on either side of the linguistic boundary (Table2). The only exception is represented by the groups located on either side of the fifth segment, making it meaningless to compute the da statistic in that case. The values and the significance of the $\mathrm{F}_{\mathrm{CT}}$ and $\delta a$ statistics (Table2) are remarkably similar for the whole boundary and for each segment, except for segment3. In this case, the two statistics give contradictory results; the value of $\delta a$ is high and significant, but the $F_{\text {CT }}$ statistic is not significant at the $5 \%$ level. This difference seems due to the lack of applicability of our regression model to the inter-group distances. The slope computed within each group population $\left(b^{\text {intra }}\right)$ is always found to be significantly different from an unconstrained slope $b^{\text {inter: }}$ the $95 \%$ confidence intervals of the unconstrained slopes $b^{\text {intra }}$ and $b^{\text {inter }}$ estimated by bootstrap were never found to overlap (see Table2 and Figure4).

The values of the statistics associated with the first three segments (Figure3, Table2) superimposed on the Mediterranean sea show that this ecological barrier does not act as a major isolating factor, in agreement with previous studies. ${ }^{20}$ In the Middle East (segment4), the linguistic groups are significantly differentiated despite the absence of apparent ecological barriers. The strongest boundary separates the Afro-Asiatic populations of East Africa and the Indo-European populations of south-western Asia and India.

\section{Discussion}

In this paper, we examined linguistic boundaries for their impact on the genetic differentiation of populations located on different sides of the boundary. We propose a new statistic $\delta a$ to quantify the additional genetic distances that can be attributed to the boundary under an IBD model. Simulations 


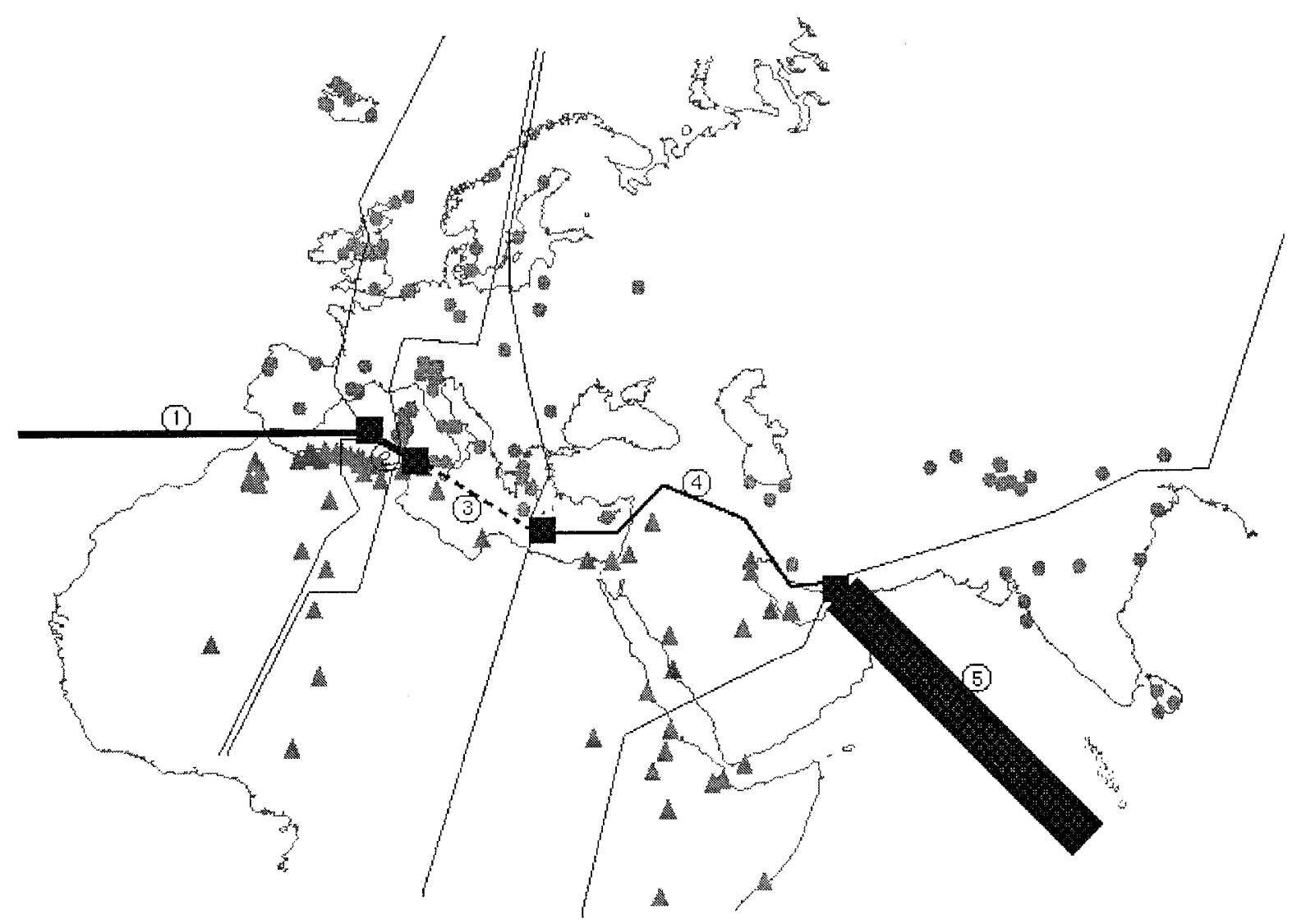

Figure 3 Location and evaluation of the linguistic boundary between populations of Afro-Asiatic and Indo-European linguistic families tested for the RH system. The width of each boundary segment is drawn proportional to the $F_{\mathrm{CT}}$ computed for this segment. If the $\mathrm{F}_{\mathrm{CT}}$ statistic is not significant at the $5 \%$ level, the segment is represented as a dashed line. The lines crossing the boundary delineate the groups of populations associated to each segment.

Table 2 Results of the evaluation of the genetic impact of the linguistic boundary between populations of the Afro-Asiatic (A) and Indo-European (B) linguistic families tested for the RH system

\begin{tabular}{|c|c|c|c|c|c|c|}
\hline & $\begin{array}{l}\text { Afro-Asiatic } \\
\text { determined } \\
\text { coefficient }(\%)^{a}\end{array}$ & $\begin{array}{l}\text { Indo-European } \\
\text { determination } \\
\text { coefficient }(\%)^{\mathrm{a}}\end{array}$ & IBD inter & $b^{\text {inter }} \neq b^{\text {intra }}$ & $\mathrm{F}_{\mathrm{CT}}$ & $\delta a$ \\
\hline Whole boundary & $8.6^{\text {**** }}$ & $11.4^{* * * *}$ & $\mathrm{Y}$ & $\mathrm{Y}$ & $0.050^{* * * *}$ & $0.075^{\text {*a*k }}$ \\
\hline Segment 1 & $21.0^{* * *}$ & $31.1^{* k}$ & Y & $\dot{Y}$ & $0.064 * * *$ & $0.066^{\text {*** }}$ \\
\hline Segment 2 & $61.7^{*}$ & 2.2 & Y & Y & $0.044^{* * * *}$ & 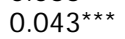 \\
\hline Segment 3 & $21.2^{*}$ & $2.5^{*}$ & $Y$ & Y & 0.008 & $0.128^{\text {*atk }}$ \\
\hline Segment 4 & $2.6 *$ & $6.8^{\text {*1*k }}$ & Y & Y & $0.016 *$ & $0.016 *$ \\
\hline Segment 5 & 0.1 & 0.7 & $\mathrm{n} / \mathrm{a}$ & $\mathrm{n} / \mathrm{a}$ & $0.281 * * *$ & $\mathrm{n} / \mathrm{a}$ \\
\hline
\end{tabular}

${ }^{*} 0.01<\mathrm{P}<0.05 ;{ }^{* * 0} 0.001<\mathrm{P}<0.01 ; * * \mathrm{*}<0.001 ; \mathrm{n} / \mathrm{a}$ : not available; aProportion of genetic variance explained by geography, obtained as the squared correlation coefficient between genetic and geography distance matrices.

show that this new statistic identifies the effect of putative barriers to geneflow more precisely than $\mathrm{F}_{\mathrm{CT}}$. However, the $\delta a$ statistic is only meaningful if the assumption of IBD holds both within and between groups of populations. Under this assumption, the additional genetic distance observed between populations located on different sides of the boundary could be attributed to reduced gene flow across the boundary, thus enhancing genetic divergence between the groups.

It seems therefore possible to understand the origin of a linguistic boundary, and to differentiate an active barrier from a mere boundary resulting from a secondary contact, by computing the $\delta a$ statistic and verifying that the assumptions of IBD are met both within and between groups. We can 


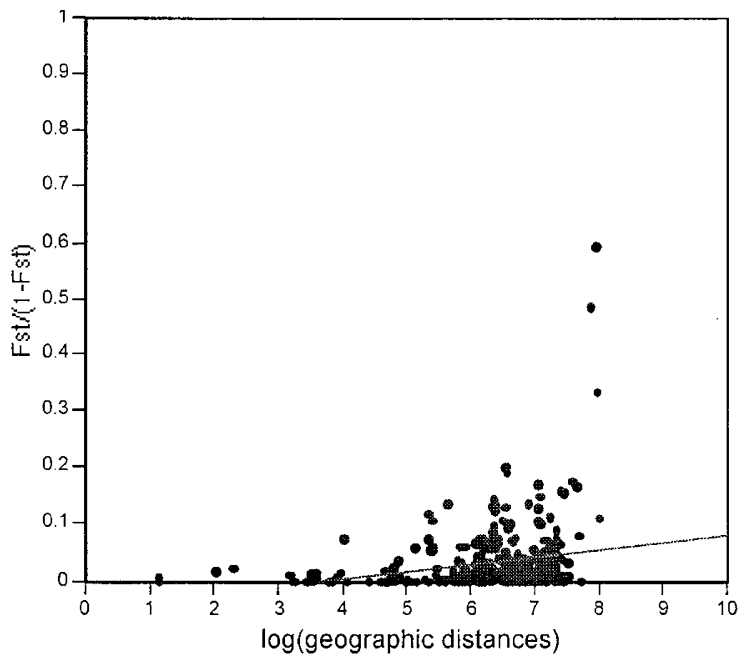

Intra-group regression

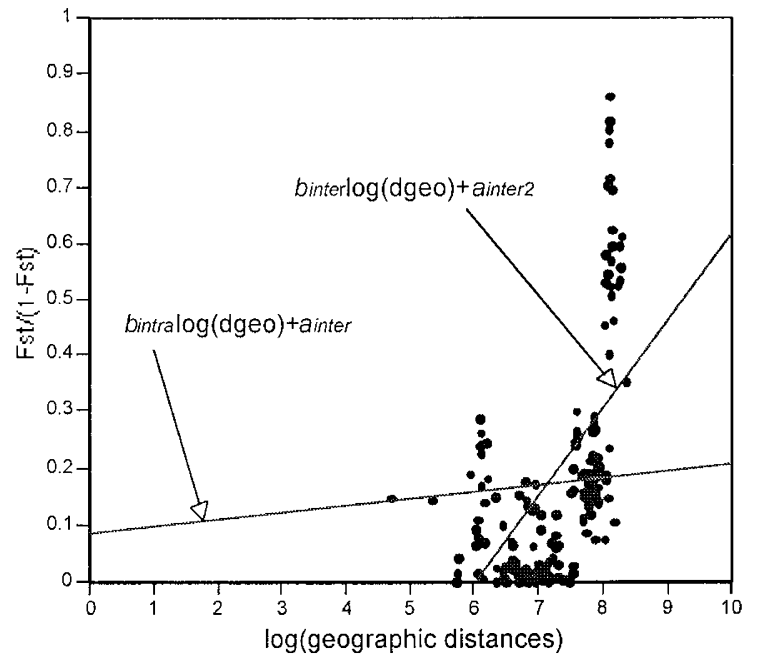

Inter-group regression

Figure 4 Regression of $\mathrm{F}_{\mathrm{ST}} /\left(1-\mathrm{F}_{\mathrm{ST}}\right)$ on the logarithm of geographic distances between populations located on the same side of the third segment defined on Figure 3 and between populations located on different sides. In the latter case, both constrained and unconstrained regressions are shown. The slopes of these two regressions are found significantly different by a bootstrap procedure (see text).

therefore envision three different outcomes of such an analysis:

(1) the $\delta a$ statistic is not significant, implying that the boundary does not act in reducing gene flow or that it is too recent to have resulted in measurable population differentiation;

2) the $\delta a$ statistic is significant, but either the IBD model is rejected when comparing populations from different groups or the slopes of the within-group and betweengroups regressions are different. In both cases, the necessary assumptions are not met; suggesting that differences between groups are due to secondary contacts;

3) the $\delta a$ statistic is significant, and the IBD model is accepted both within and between groups, suggesting that the boundary actually contributes to prevent gene flow.

The computation of the $\mathrm{F}_{\mathrm{CT}}$ statistic and its comparison with $\delta a$ can also be useful in understanding the evolutionary forces at work in a given region. Simulations show that the $\mathrm{F}_{\mathrm{CT}}$ statistic can reveal significant differences between groups of populations in the absence of any boundary but in the presence of IBD (case3, Table1). In most cases, the comparison of the $F_{C T}$ to $\delta a$ should allow us to resolve this ambiguity.

We see that no single summary statistic is enough to understand fully the processes involved in the origin and the maintenance of a genetic barrier. The approach we advocate here is thus plural. The results obtained using different statistics should be compared with each other, and the assumptions underlying the IBD model should always be verified and tested.

Several features of the linguistic boundary between speakers of Indo-European and Afro-Asiatic languages seem to emerge. First, the effect of the boundary on genetic distances seems quite heterogeneous, especially in the middle section of the boundary. The Mediterranean Sea does not seem to represent a strong barrier to gene flow, as is documented for historical times, with the development of commercial routes between the northern and southern part of the Mediterranean Sea. ${ }^{28,29}$ Second, the present linguistic boundary seems mainly to be the result of a secondary contact between two differentiation centres, rather than being an obstacle to gene flow between the different sides of the boundary. This interpretation is supported by the fact that observed IBD within linguistic groups does not hold between groups (Table2). The present approach could be applied to other linguistic boundaries to see if they also result from secondary contacts, as previously suggested. ${ }^{4,14,30,31}$

Although our method has been devel oped to study linguistic boundaries between human populations, it could be used to study the impact of other types of boundary in different organisms. The biological interpretation of the $\delta a$ statistic in terms of reduced gene flow through a boundary would certainly better apply to physical barriers like mountains, rivers or deserts. The linear regression model we have used here to quantify the influence of a given boundary permits incorporation of spatial constraints when comparing the genetic constitution of two groups of populations. More realistic models could certainly be envisioned. For instance, it 
would certainly be more realistic to assume that the boundary has more influence on populations in its vicinity, and thus to use a model with a decreasing effect of the boundary with geographic distance.

\section{Acknowledgements}

We are grateful to Alicia Sanchez-Mazas for providing access to her genetic database. This work has been supported by Swiss FNRS grants 31-39847.93 and 31-054059.98 to AL. A program to compute and test the different statistics presented here is available from Isabelle Dupanloup de Ceuninck on request.

\section{References}

1 Wright S: Isolation by distance. Genetics 1943; 28: 114-138.

2 Malécot G: Les mathématiques de l'hérédité. Masson: Paris, 1948.

3 Morton NE: Isolation by distance. In Morton Ne (ed.). Genetic Structure of Populations. University of Hawaii Press: Honolulu, 1973, pp 76-77.

4 Barbujani G, Sokal RR: Genetic population structure of Italy. I. Geographic patterns of gene frequencies. Hum Biol 1991; 63: 253-272.

5 Barbujani G, Pilastro A: Genetic evidence on origin and dispersal of human populations speaking languages of the Nostratic macrofamily. Proc Nat Acad Sci USA 1993; 90: 4670-4673.

6 Excoffier L, Harding RM, Sokal RR, Pellegrini B, Sanchez-M azas A: Spatial differentiation of Rhesus and GM haplotype frequencies in Sub-Saharian Africa and its relation to linguistic affinities. Hum Biol 1991; 63: 273-307.

7 Cavalli-Sforza LL, Menozzi P, Piazza A: The History and Geography of Human Genes. Princeton University Press: Princeton, NJ, 1994.

8 Poloni ES, Excoffier L, Mountain JL, Langaney A, Cavalli-Sforza LL: Nuclear DNA polymorphism in a Mandenka population from Senegal: comparison with eight other human populations. Ann Hum Genet 1995; 59: 43-61.

9 Poloni ES, Semino O, Passarino G, Santachiara-Benerecetti AS, Dupanloup I, Langaney A, Excoffier L: Human genetic affinities for Y-chromosome P49a,f/Taql haplotypes show strong correspondence with linguistics. Am । Hum Genet 1997; 61: 1015-1035.

10 Cavalli-Sforza LL, Piazza A, Menozzi P, Mountain J: Reconstruction of human evolution: Bringing together genetic, archaeological and linguistic data. Proc Nat Acad Sci USA 1988; 85: 6002-6006.

11 Cavalli-Sforza LL, Minch E, Mountain JL: Coevolution of genes and languages revisited. Proc Nat Acad Sci USA 1992; 89: 5620-5624.

12 Greenberg JH, Turner CG, Zegura SL: The settlement of the Americas: a comparison of the linguistic, dental and genetic evidence. Curr Anthropol 1986; 27: 477-497.

13 Excoffier L, Pellegrini P, Sanchez-Mazas A, Simon C, Langaney A: Genetics and history of Sub-Saharan Africa. Yearbook Phys Anthropol 1987; 30: 151-194.
14 Barbujani G, Sokal RR: Zones of sharp genetic change in Europe are also linguistic boundaries. Proc Nat Acad Sci USA 1990; 87: 1816-1819.

15 Sokal RR, Oden NL, Thomson BA: Genetic changes across language boundaries in Europe. Am J Phys Anthropol 1988; 76: 337-361.

16 Sokal RR, Oden NL, Legendre P, Fortin MJ, Kim J, Vaudor A: Genetic differences among language families in Europe. Am J Phys Anthropol 1989; 79: 489-502.

17 Barbujani G, Jacquez GM, Ligi L: Diversity of some gene frequencies in European and Asian populations. V. Steep multilocus clines. Am J Hum Genet 1990; 47: 867-875.

18 Barbujani G, Oden NL, Sokal RR: Detecting regions of abrupt change in maps of biological variables. System Zool 1989; 38 376-389.

19 Stenico M, Nigro L, Barbujani G: Mitochondrial lineages in Ladinspeaking communities of the eastern Alps. Proc Roy Soc Lond Series B: Biol Sci 1998; 265: 555-561.

20 Simoni L, Gueresi P, Pettener D, Barbujani G: Patterns of gene flow inferred from genetic distances in the Mediterranean region. Hum Biol 1999; 71: 399-415.

21 Voronoi MG: Nouvelles applications des paramètres continus à la théorie des formes quadratiques, deuxième mémoire, recherche sur les paralléloedres primitifs. J Reine Angew Math 1908; 134: 198-287.

22 Excoffier L, Smouse P, Quattro JM: Analysis of molecular variance inferred from metric distances among DNA haplotypes: application to human mitochondrial DNA restriction data. Genetics 1992; 131: $479-491$.

23 Schneider S, Kueffer JM, Roessli D, Excoffier L: Arlequin ver. 1.1: A software for population genetic data analysis. Genetics and Biometry Laboratory, University of Geneva, Switzerland, 1997.

24 Rousset F: Genetic differentiation and estimation of gene flow from F-statistics under isolation by distance. Genetics 1997; 145: 1219-1228.

25 Mantel NA: The detection of disease clustering and a generalized regression approach. Cancer Res 1967; 27: 209-220.

26 Hudson RR: Gene genealogies and the coalescent process. In Futuyma D, Antonovics J (eds). Oxford Surveys in Evolutionary Biology. vol. 7, Oxford University Press: Oxford, 1990; pp 1-44.

27 Sanchez-M azas A: Polymorphisme des systèmes immunologiques Rhésus, GM et HLA et histoire du peuplement humain. Genetics and Biometry Laboratory: University of Geneva, Switzerland, 1990

28 Hetzron R: Afro-Asiatic languages. In Comrie B (ed.). The World's Major Languages. Croom Helm: London; Sydney, 1987; pp 645-653.

29 Gamkrelidze TV, Ivanov VV: Indo-European and the Indo-Europeans. Mouton de Gruyter: Berlin; New York, 1995.

30 Barbujani G, Vian P, Fabbris L: Cultural barriers associated with large gene frequency differences among Italian populations. Hum Biol 1992; 64: 479-495.

31 Renfrew C: World linguistic diversity. Scient Amer 1994; 104-110. 